\title{
Post traumatic isolated multiple cranial nerve palsies
}

\section{Sunil Munakomi*}

Department of Neurosurgery, College of medical sciences, Nepal

A sixty-five years male patient presented in our neurological clinic following a fall from a slope while grazing grass in remote Nepal. He was complaining of drooping of the right eye lid and also having double vision. His Glasgow coma scale (GCS) was 15/15 and his vital parameters were within normal range. Following thorough neurological examination, he was found to have bilateral third (right $>$ left), bilateral fourth (right $>$ left) and partial right sixth nerve palsies along with loss of convergence (Figure 1). Computerized tomogram (CT) head however did not reveal any findings so as to localize the cause for the bilateral ophthalmoplegia. Magnetic resonance imaging (MRI) T2W and the FLAIR (Fluid attenuated inverse recovery) sequences revealed focal contusion in the anterio-lateral aspect of the midbrain along the region of the pyramidal tracts within the crus cerebri on the right side (Figure 2). Lesions in the brainstem characteristically present

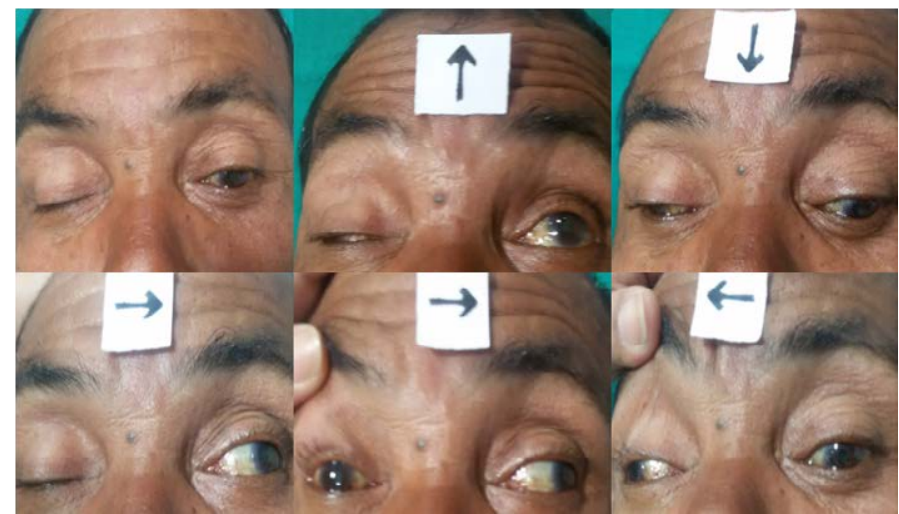

Figure 1. Clinical image showing post traumatic ophthalmoplegia involving multiple cranial nerves.

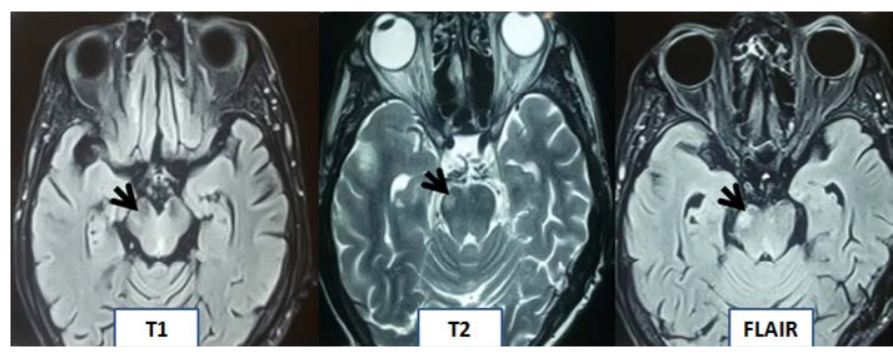

Figure 2. MRI findings showing signal changes in the pons which helps to localize the cause of the ophthalmoplegia within the brainstem.

Copyright: (02016 Munakomi S. This is an open-access article distributed under the terms of the Creative Commons Attribution License, which permits unrestricted use, distribution, and reproduction in any medium, provided the original author and source are credited. with features of crossed hemiplegia. However, our patient did not have even soft pyramidal signs, making the localization even more difficult. Bilateral ophthalmoplegia due to peripheral cause is a rare entity. Due to the involvement of third, fourth and sixth cranial nerves, we ruled out the possibility of the lesions in the cavernous sinus or that of the orbits by performing MRI studies of the corresponding regions as well.

The patient was managed conservatively and was discharged on tapering doses of oral steroid (Prednisolone) therapy. There was minimal improvement on his spontaneous eyelid elevation on the right sight during his follow up at first month.

Post traumatic multiple cranial nerve palsies are a well reported entity [1]. In our case, there was a focal lesion in the midbrain accounting for the probable central cause for the bilateral ophthalmoplegia. In circumstances wherein the imaging modalities do not reveal any localizing pathology, neuropraxia following accelerating and decelerating injury, is the most attributable cause. Moreover, the imaging findings do not correlate well with the clinical findings in many circumstances [2]. The recovery is variable and depends upon the severity of initial injury [3].

\section{References}

1. Keane JR, Baloh RW. Post-traumatic cranial neuropathies. In: Evans RW, Ed. The Neurology of Trauma. Philadelphia: Saunders; 1992: 849e868.

2. Dhaliwal A, West AL, Trobe JD, Musch DC (2006)Third, fourth and sixth cranial nerve palsies following closed head injury. J Neuroophthalmol 26: 4-10.[Crossref]

3. Coello AF, Canals AG, Gonzalez JM, Martín JJ (2010)Cranial nerve injury after minor head trauma. J Neurosurg 113: 547-555. [Crossref]

Correspondence to: Sunil Munakomi, Department of Neurosurgery, College of medical sciences, Nepal, E-mail: sunilmunakomi@gmail.com

Received: December 04, 2016; Accepted: December 13, 2016; Published: December 15, 2016 